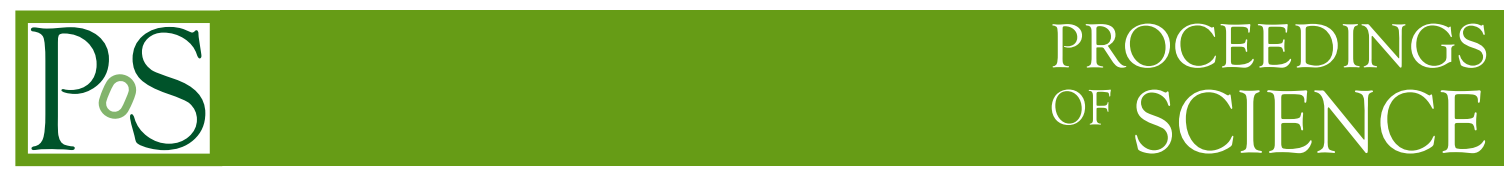

\title{
THE SOFTWARE FRAMEWORK OF THE BELLE II SILICON VERTEX DETECTOR AND ITS DEVELOPMENT FOR THE 2016 TEST-BEAM AT DESY
}

G. Caria ${ }^{* \dagger a}$, K. Adamczyk ${ }^{r}$, H. Aihara ${ }^{o}$, C. Angelini ${ }^{h, i}$, T. Aziz ${ }^{g}$, V. Babu ${ }^{g}$, S. Bacher ${ }^{r}$, S. Bahinipati ${ }^{d}$, E. Barberio ${ }^{a}$, Ti. Baroncelli ${ }^{a}$, To. Baroncelli ${ }^{a}$, A. K. Basith ${ }^{e}$, G. Batignani $^{h, i}$, A. Bauer ${ }^{b}$, P. K. Behera ${ }^{e}$, T. Bergauer $^{b}$, S. Bettarini ${ }^{h, i}$, B. Bhuyan ${ }^{f}$, T. Bilka ${ }^{c}$, F. Bosi ${ }^{i}$, L. Bosisio ${ }^{j}, k$, A. Bozek ${ }^{r}$, F. Buchsteiner ${ }^{b}$, L. Bulla ${ }^{b}$, G. Casarosa $^{i}$, M. Ceccanti ${ }^{i}$, D. Červenkov ${ }^{c}$, S. R. Chendvankar ${ }^{g}$, N. Dash ${ }^{d}$, G. De Pietro ${ }^{h, i}$,

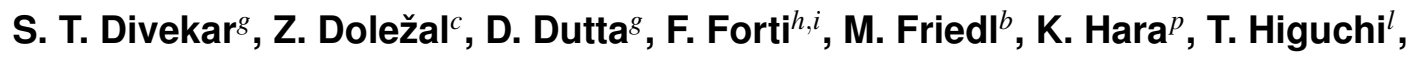
T. Horiguchi ${ }^{n}$, C. Irmler ${ }^{b}$, A. Ishikawa ${ }^{n}$, H. B. Jeon ${ }^{q}$, C. Joo ${ }^{l}$, J. Kandra ${ }^{c}$, N. Kambara ${ }^{p}$, K. H.Kang ${ }^{q}$, T. Kawasaki ${ }^{C, m}$, P. Kodyš ${ }^{c}$, T. Kohriki ${ }^{p}$, S. Koike ${ }^{D, p}$, M. M. Kolwalkar ${ }^{g}$, R. Kumar ${ }^{s}$, W. Kun ${ }^{o}$, P. Kvasnička ${ }^{c}$, C. La Licata ${ }^{j, k}$, L. Lanceri ${ }^{j, k}$, J. Lettenbicher ${ }^{b}$, J. Libby ${ }^{e}$, T. Lueck ${ }^{h, i}$, M. Maki $^{p}$, P. Mammini ${ }^{i}$, S. N. Mayekar ${ }^{g}$, G. B. Mohanty ${ }^{g}$, S. Mohanty ${ }^{A, g}$, T. Morii ${ }^{l}$, K. R. Nakamura ${ }^{p}$, Z. Natkaniec ${ }^{r}$, Y. Onuki ${ }^{o}$, W. Ostrowicz ${ }^{r}$, A. Paladino ${ }^{h, i}$, E. Paoloni ${ }^{h, i}$, H. Park ${ }^{q}$, F. Pilo ${ }^{i}$, A. Profeti ${ }^{i}$, I. Rashevskaya ${ }^{B, k}$, K. K. Rao ${ }^{g}$, G. Rizzo ${ }^{h, i}$, Resmi P. K. ${ }^{e}$, M. Rozanska ${ }^{r}$, J. Sasaki ${ }^{o}$, N. Sato ${ }^{p}$, S. Schultschik ${ }^{b}$, C. Schwanda ${ }^{b}$, Y. Seino $^{m}$, N. Shimizu $^{o}$, J. Stypula $^{r}$, J. Suzuki ${ }^{p}$, S. Tanaka ${ }^{p}$, G. N. Taylor ${ }^{a}$, R. Thalmeier ${ }^{b}$, R. Thomas ${ }^{g}$, T. Tsuboyama ${ }^{p}$, S. Uozumi ${ }^{q}$, P. Urquijo ${ }^{a}$, L. Vitale ${ }^{j, k}$, S. Watanuki ${ }^{n}$, M. Watanabe ${ }^{l}$, I. J. Watson ${ }^{o}$, J. Webb $^{a}$, J. Wiechczynski ${ }^{r}$, S. Williams ${ }^{a}$, B. Würkner ${ }^{b}$, H. Yamamoto ${ }^{n}$, H. Yin ${ }^{b}$, T. Yoshinobu ${ }^{p}$, L. Zani ${ }^{h, i}$ (Belle II SVD Collaboration) 
${ }^{a}$ School of Physics, University of Melbourne, Melbourne, Victoria 3010, Australia

${ }^{b}$ Institute of High Energy Physics, Austrian Academy of Sciences, 1050 Vienna, Austria

${ }^{c}$ Faculty of Mathematics and Physics, Charles University, 12116 Prague, Czech Republic

${ }^{d}$ Indian Institute of Technology Bhubaneswar, Satya Nagar, India

${ }^{e}$ Indian Institute of Technology Madras, Chennai 600036, India

${ }^{f}$ Indian Institute of Technology Guwahati, Assam 781039, India

${ }^{g}$ Tata Institute of Fundamental Research, Mumbai 400005, India, ${ }^{A}$ also at Utkal University, Bhubaneswar 751004, India

${ }^{h}$ Dipartimento di Fisica, Universita ' di Pisa, I-56127 Pisa, Italy

${ }^{i}$ INFN Sezione di Pisa, I-56127 Pisa, Italy

${ }^{j}$ Dipartimento di Fisica, Universita' di Trieste, I-34127 Trieste, Italy

${ }^{k}$ INFN Sezione di Trieste, I-34127 Trieste, Italy, ${ }^{B}$ presently at TIFPA - INFN, I-38123 Trento, Italy

${ }^{l}$ Kavli Institute for the Physics and Mathematics of the Universe (WPI), University of Tokyo, Kashiwa 277-8583, Japan

${ }^{m}$ Department of Physics, Niigata University, Niigata 950-2181, Japan, ${ }^{C}$ presently at Kitasato University, Sagamihara 252-0373, Japan

${ }^{n}$ Department of Physics, Tohoku University, Sendai 980-8578, Japan

${ }^{\circ}$ Department of Physics, University of Tokyo, Tokyo 113-0033, Japan

${ }^{p}$ High Energy Accelerator Research Organization (KEK), Tsukuba 305-0801, Japan, ${ }^{D}$ deceased

${ }^{q}$ Department of Physics, Kyungpook National University, Daegu 702-701, Korea

${ }^{r}$ H. Niewodniczanski Institute of Nuclear Physics, Krakow 31-342, Poland

${ }^{s}$ Punjab Agricultural University, Ludhiana 141004, India

The Silicon Vertex Detector of Belle II will be fundamental not only for the reconstruction of B meson vertices, but also for the reconstruction of neutral particles like $K_{S}$, and the tracking of low- $p_{t}$ particles like slow pions associated with the decays of the abundant $D^{*}$ mesons. As a consequence great importance is given to the performance of the software that deals with the simulation and reconstruction of the SVD events. The Belle II experiment is the successor to Belle, one of the two experiments that first observed CP violation in the decay of B mesons. The SuperKEKB collider will deliver 40 times more luminosity than its predecessor KEKB, which requires major upgrades to the detector hardware. This also poses new challenges not only for the data collection and storage, but also for the software framework which is used to process and analyse the experimental data. In this article we present in detail the SVD software framework together with its development for the test of the Vertex Detector system, which took place at DESY in April 2016 using an electron beam.

The 25th International workshop on vertex detectors

September 26-30, 2016

La Biodola, Isola d'Elba, ITALY

\footnotetext{
*Speaker.

${ }^{\dagger}$ Email: gcaria@student.unimelb.edu.au
} 


\section{The Silicon Vertex Detector of Belle II}

The Belle detector [1], operated at the KEKB electron-positron collider located at Tsukuba, Japan, has accumulated a data sample of $1000 \mathrm{fb}^{-1}$ by the end of 2010, allowing, among the other results, for the observation of CP violation in the decay of B mesons [2] which led to the 2008 Nobel Prize being awarded to the theorists Kobayashi and Maskawa [3].

The KEKB collider is being upgraded to SuperKEKB [4], with the aim to have a luminosity 40 times larger than before. Additionally, the Belle II detector will replace the old Belle detector. This experiment is characterized as a B-factory, given that its goal is to produce large numbers of $B / \bar{B}$ meson pairs, which are very suitable candidates for the observation of $\mathrm{CP}$ violation and other phenomena.

The innermost component of the Belle II detector, the VerteX Detector (VXD), is responsible for the reconstruction of the vertices of the decaying particles, which provides essential information in the study of time-dependent CP violation. The Belle II detector includes two upgrades for the VXD: a very precise and high-granularity double-layer PiXel Detector (PXD) and a new Silicon Vertex Detector (SVD) with 4 layers of silicon sensors [5]. With respect to the previous SVD, the new one is shifted outward to a maximum radius of $140 \mathrm{~mm}$ in order to make space for the PXD, which consequently increases the total spatial coverage provided by the VXD. Special features of the SVD are the ability to reconstruct the decays of neutral particles like $K_{S}$ that do not deposit energy while passing through the PXD, and the possibility to track low- $p_{t}$ particles like the slow pions produced in the decay of $D^{*}$ mesons that do not reach the CDC. Furthermore, the SVD provides very precise hit timing information and implements the use of the energy loss information in the sensor for better hit selection and discrimination (this feature was not present in the Belle SVD).

The SVD is made of double-sided silicon strip sensors, whose arrangement is shown in Figure 1. Double-sided sensors provide two coordinates of the hits (with the $z$ coordinate being defined by the sensor position). The two directions of the strips are known as $\mathrm{U}$ (parallel to the beam direction, with a small pitch $p$ ) and $\mathrm{V}$ (perpendicular to beam direction, i.e. radial, with a large pitch $n$ ). The innermost SVD layer (i.e. layer 3 of the VXD) has small rectangular sensors (thickness: $320 \mu \mathrm{m}$ ). The other layers are composed of two types of sensors: large rectangular sensors (named barrel sensors, $320 \mu \mathrm{m})$ and slanted sensors $(300 \mu \mathrm{m})$ with a trapezoidal shape, which improves acceptance and precision for forward boosted particles and allows to extend the polar angle coverage to $\left[17^{\circ}, 150^{\circ}\right]$.

\section{The SVD Software Framework}

The SVD software framework is part of the Belle II analysis and simulation framework (basf2) and as such inherits its specific characteristics. The SVD software deals with the simulation of the interaction of particles with the SVD (implementation of the SVD geometry into the detector simulation, the simulation of the SVD sensors response) and the reconstruction of SVD hits, either from real data or from MC simulation (decoding of raw data and treatment of strip signals). The following part of this section deals with the explanation of SVD software algorithms. 

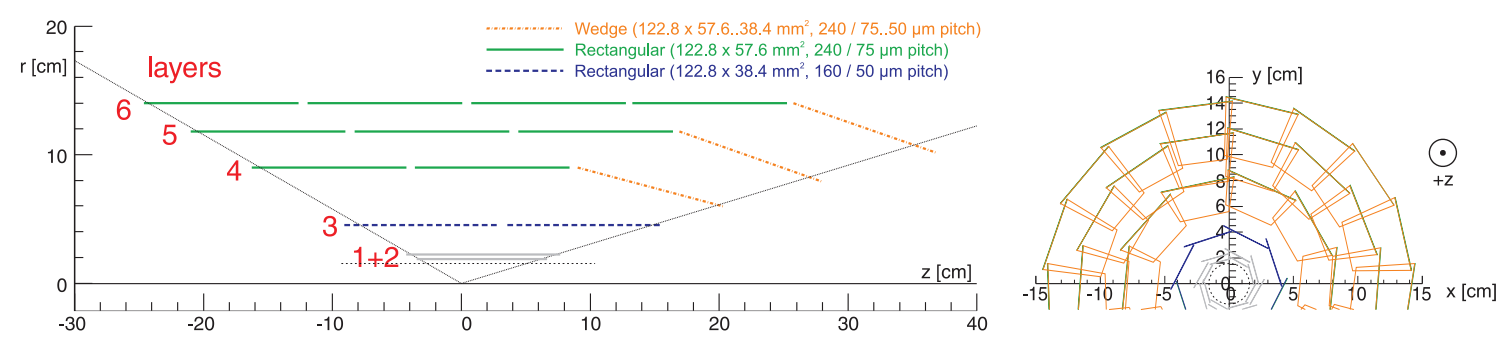

Figure 1: The 6 layers of the VXD system with emphasis on the SVD, whose cross sections are shown in the $r-z$ plane (left), and the $x-y$ plane (right, only top part). The different dimensions of the three types of sensors are annotated in the left picture [5].

The electron-hole pairs produced by the passage of a charge particle through an SVD sensor are collected by the strips and then read-out by APV25 chips, which sample the charge deposition creating so-called raw data. The SVD unpacker then decodes the raw data and transforms them into digits. The SVD digit sorter algorithm organises digits in such a way that each strip will be assigned a specific charge and hit time, and it also ensures digits are arranged in a spatial order in preparation for the hit reconstruction. To achieve this, strip signals have to be mapped to corresponding physical strips within each sensor. The deposited charge is normally shared among a number of strips and for this reason, the SVD clusterizer algorithm scans each SVD sensor and deals with the grouping of strips to form SVD clusters. Only meaningful strips are selected in this process, while fake hits and random noise are neglected through the use of clustering thresholds, as will be elaborated later. Tables of known defective strips are used to filter out this source of error in the reconstruction. The presence of a magnetic field is considered, given that it causes a deflection of the charge flow inside the sensors and a systematic shift in the charge collection position. Finally, a position and relative uncertainty is assigned to the cluster with the aid of dedicated algorithms, such as the Center Of Gravity algorithm, generally used for small size clusters, and the Analog Head Tail algorithm, used for large clusters. The combination of clusters on both sides of each sensor (i.e. a pair of $x, y$ coordinates for each hit) is now ready to be given as input to the tracking algorithms. The SVD clusterizer provides two important variables for each cluster: a reliable time measurement, which can be used in the discrimination against off-time beam background hits, and a value for the energy deposited in the hit, which enables the identification of the particle passing through the sensor. The various steps of the SVD software framework are schematically summarized in Figure 2.

\section{Absolute Charge Calibration}

The output of the strip charge readout is given in Analog to Digital Converter counts. As such, this quantity has a value which does not explicitly provide any physical information about the hit that caused the charge deposition in the sensor.

In order to extract useful information about the charge deposition event that takes place in the sensor, we need to know the number of electron-hole pairs created which, combined with knowledge of the ionization energy of the material, corresponds to the energy deposited in the sensor. In other words, we can gain physical information about energy loss in the sensors only if 


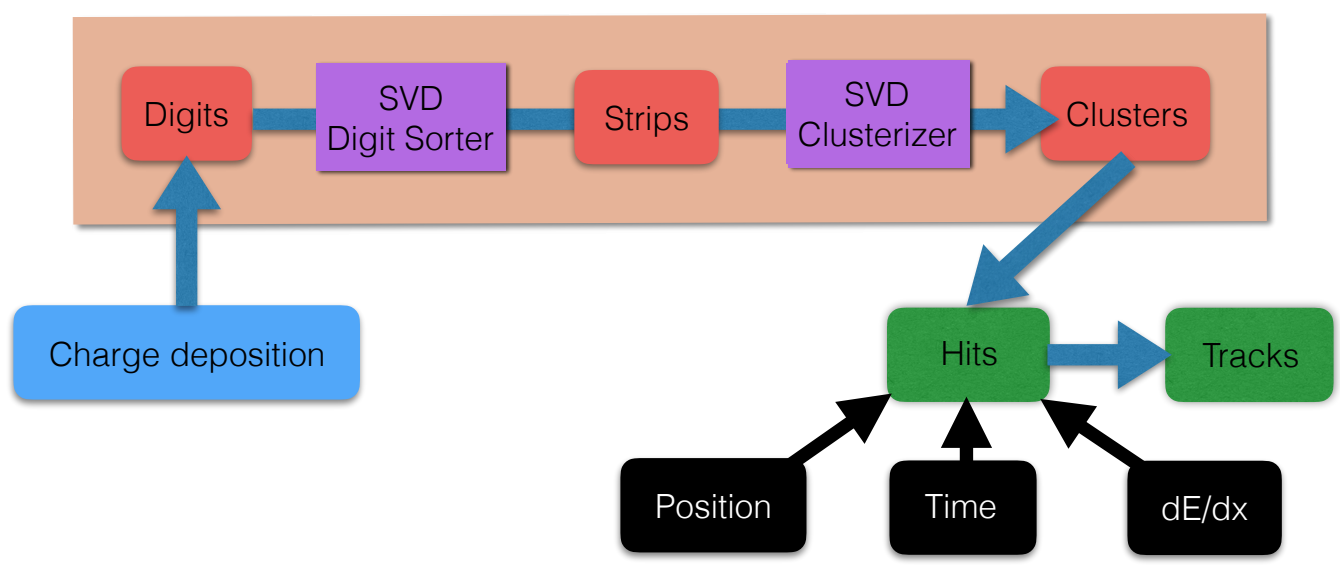

Figure 2: This schematic shows how the SVD software framework (highlighted pink region) plays the role of filling the gap between the production of hits in the sensors and the creation of tracks composed by these hits.

calibration constants that allow for the conversion from ADC counts to the number of electron-hole pairs produced in the sensors are used.

We developed an algorithm that provides these calibration constants, for different scenarios:

- Traversing particles that are heavier than electrons;

- Traversing electrons, as was the case for the DESY '16 test-beam.

The latter case has been created specifically to be applied to the experimental data produced by test-beam. The type of calibration performed by this algorithm is absolute: it includes all the processes that the place when a charged particle traverses an SVD sensor, including the ionization in the sensor, the charge deposition and the charge readout.

\subsection{Calibration with particles heavier than electrons}

This algorithms is used in scenarios where data is taken with charged particles heavier than electrons and loss information is in the form of $\mathrm{ADC} / \mathrm{cm}$, as it is the case for cosmic muons. When the energy of the incoming particle is known, it is possible to build a distribution of experimentally measured energy loss vs momentum, which can then be compared to theoretical models in order to extract calibration contants. For particles heavier than electrons, the stopping power functions have been extensively studied and are well modeled. The well-known Bethe Bloch formula has been corrected to provide the Most Probable Value (MPV) and not the average value, and improved to describe energy loss in thin sensors, as is the case for the tracking detectors of Belle II. The most up-to-date theoretical description of the MPV for energy loss in thin sensors is the Landau-VavilovBichsel function [6] given as

$$
\Delta_{p}=\xi\left[\ln \frac{2 m c^{2} \beta^{2} \gamma^{2}}{I}+\ln \frac{\xi}{I}+j-\beta^{2}-\delta(\beta \gamma)\right],
$$

where $\xi=(K / 2)\langle Z / A\rangle\left(x / \beta^{2}\right) \mathrm{MeV}$ for a detector with a thickness $x$ in $\mathrm{gcm}^{-2}$ and $j=0.200$. This function includes a density correction term $\delta(\beta \gamma)$ that accounts for the flattening of the electrical 
field for high momentum particles, and for a detector of thickness $x$ scales as $\Delta_{p} / x \propto a \ln x+b$. Its behaviour is shown in Figure 3, for different sensor thickness values.

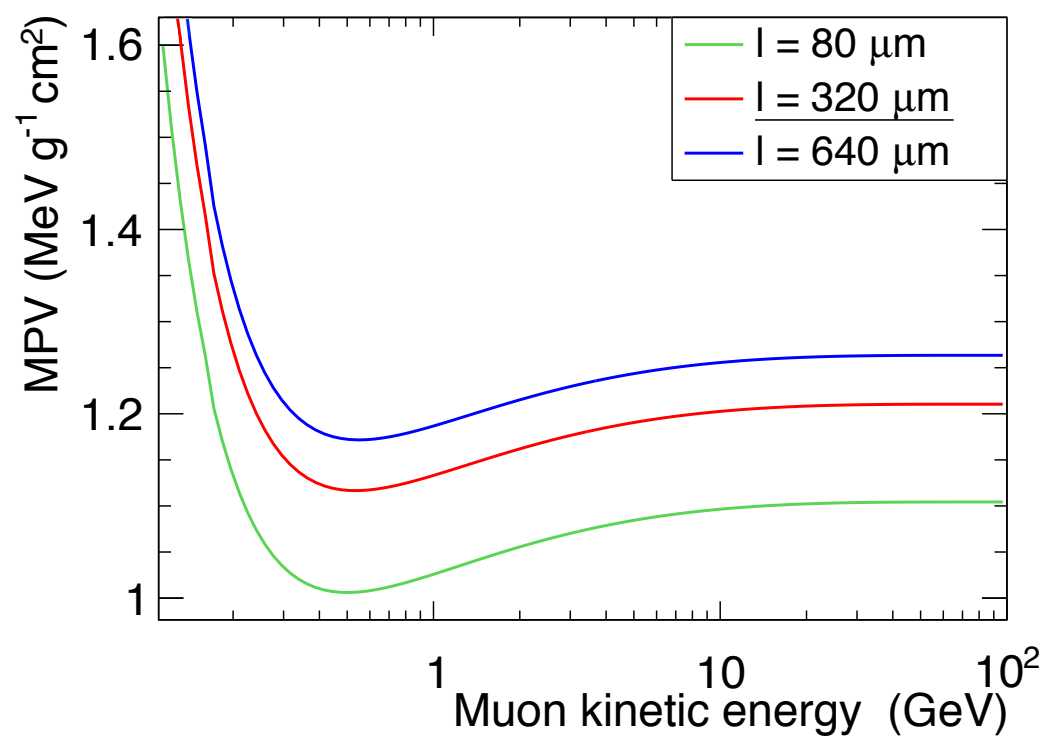

Figure 3: The Landau-Vavilov-Bichsel function used in this study is shown for different sensor thickness values, including the thickness of the Belle II SVD sensors, $320 \mu \mathrm{m}$.

The Landau-Vavilov-Bichsel fit is carried out on experimental points [MPV, p] created by this algorithm through $d E / d x$ distributions for each momentum value, extracting the MPV of the distribution using a fit to the Landau-Vavilov straggling function.

Points obtained are fitted with the Landau-Vavilov-Bichsel function using two parameters $A$ and $B$, that is:

$$
\frac{\Delta_{p[A D U]}}{l}=A+B \cdot \frac{\Delta_{p[\mathrm{MeV}]}}{l},
$$

where $\Delta_{p}$ represents the most probable energy loss, $l$ is the sensor thickness, and we expect the offset A to be consistent with zero. This allows for the extraction of the calibration constants $A$ and $B$ to convert from the experimentally measured curve in ADU/cm to theoretical predictions for $\mathrm{MeV} / \mathrm{cm}$. An example output of this procedure is shown in Figure 4.

\subsection{Calibration Using Electrons at the DESY' 16 Test-Beam}

A theoretical description of the collisional energy loss of high energy $(>1 \mathrm{GeV})$ electrons, such as those used in the DESY ' 16 test-beam setup, is not readily available in the literature. For this reason, we have chosen to extract theoretical information about this type of energy loss using the simulated data of the test-beam setup directly. This simulation is carried out with the Geant 4 toolkit [7] and is therefore highly reliable.

The MPVs for the experimental $(\alpha)$ and simulated $(\beta)$ energy loss distributions are found through a fit to the Vavilov function, and calibration constants $C$ are then obtained by a simple 


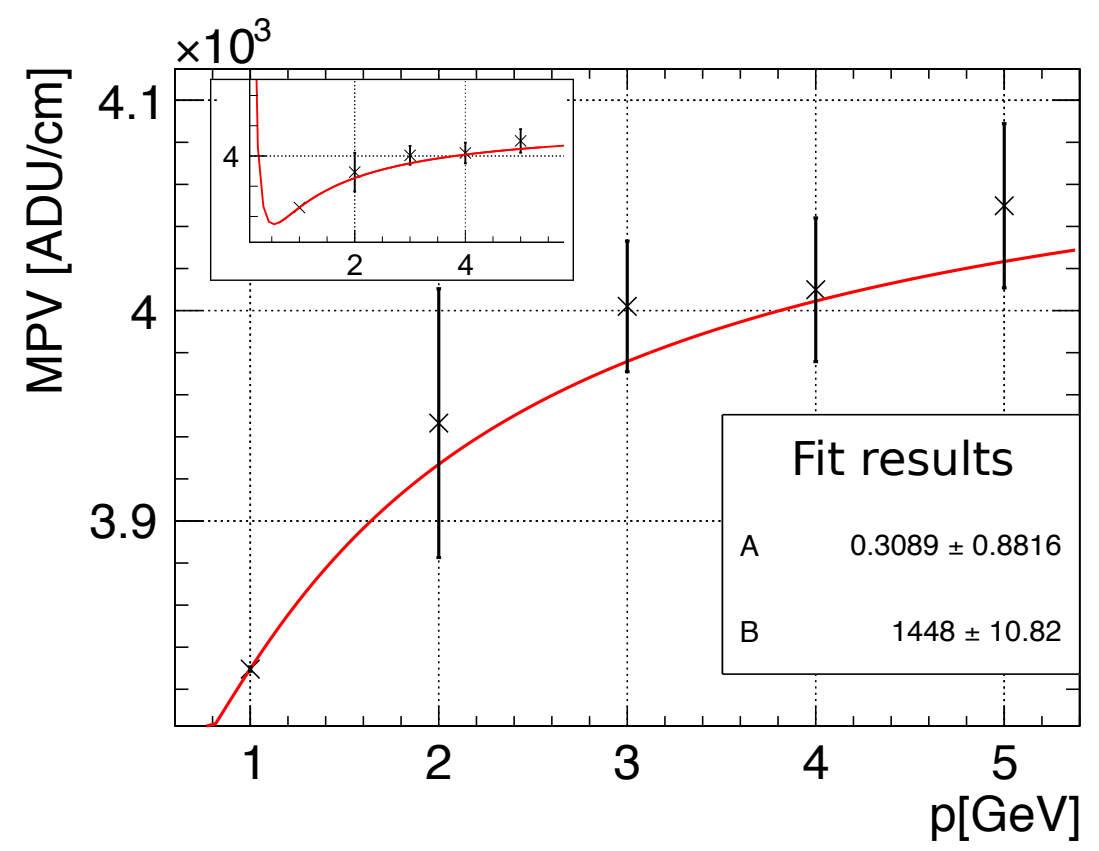

Figure 4: An example of how the Landau-Vavilov-Bichsel fits for the $M P V$ vs $p$ graph, with the fit results printed. The momentum has no uncertainty bar since it is set in the simulation. In the insert a larger portion of the $x$ and $y$ axes is shown for the same graph. The fit results A and B are defined in Equation 3.2.

comparison:

$$
C \pm \Delta C=\frac{\alpha}{\beta} \pm\left(\frac{\Delta \alpha}{\alpha}+\frac{\Delta \beta}{\beta}\right) \frac{\alpha}{\beta}
$$

\section{Clustering Efficiency and Signal to Noise Thresholds}

SVD strips are considered for the formation of clusters, only if they pass a minimum threshold on their Signal to Noise ratio, which we can define as $S N T_{\text {noise, }}$, where SNT stands for Signal to Noise Threshold. Two other thresholds are applied in the clustering algorithm: at least one strip, named the seed, has to have a Signal to Noise value higher than $S N T_{\text {seed }}$ and the whole cluster has to pass a threshold $S N T_{c l s}$, which is defined as $S N T_{c l s} \equiv \sum S_{i} / \sqrt{\sum N_{i}^{2}}$, where the sum extends to all the signal (noise) values $S_{i}\left(N_{i}\right)$ of the strips in the cluster.

These three thresholds are used to ensure only clusters related to real hits are selected as input for the tracking algorithms. Clearly these values have to be carefully selected. Indeed, if they are too high we would neglect a large fraction of meaningful hits (i.e. yielding a low clustering efficiency), while if they are too low we would have a high clustering efficiency, which comes at the price of including many random and irrelevant hits in the next stages of the reconstruction. The latter point has a fundamental relevance for the VXD of Belle II, which will operate in the high background environment of the SuperKEKB accelerator. For this reason we carried out an analysis of sets of SNT in terms of yielded clustering efficiency and fake hits rate, and applied this study to the experimental data collected at the DESY' 16 test-beam. 
In this study we first find the intersection between the reconstructed track and the sensor, then apply the clustering algorithms to search for clusters in a neighbourhood of this intersection, using all possible combinations of the chosen set of SNTs. A positive or negative output of this search defines the clustering efficiency. It is important to notice that the neighbourhood of the intersection between track and sensor is defined as twice the strip pitch of a given sensor, since a cluster that is found outside this region cannot be associated with the reconstructed track. An example output of this algorithm is shown in Figure 5.

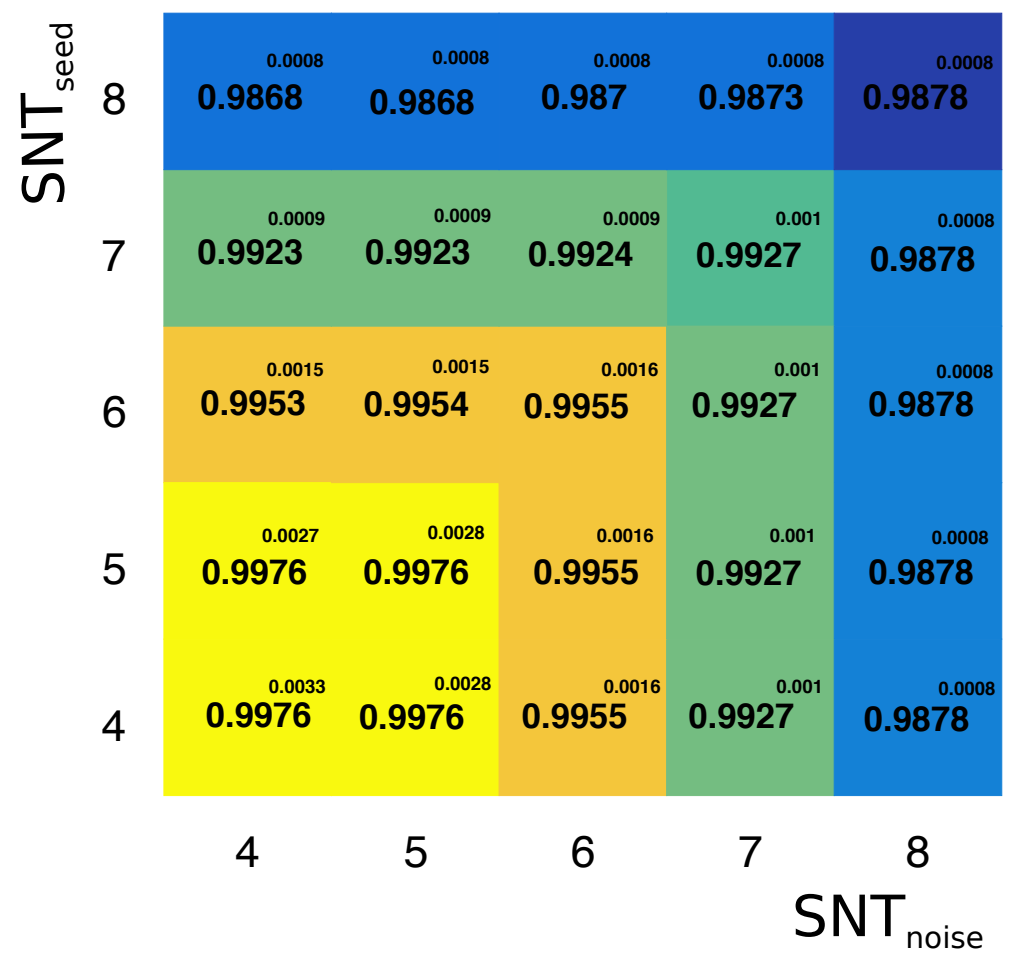

Figure 5: An example output of this study. For a fixed value of one SNT type (chosen to be the $S N T_{c l s}=2$ ), a graph of the other two variables is obtained (in this case $S N T_{\text {seed }}$ vs $S N T_{\text {noise }}$ ), representing clustering efficiency in black, with the uncertainty as superscript.

\section{Conclusion}

The central role of the SVD in the Belle II detector is properly supported by a dedicated software framework, responsible for the simulation and the reconstruction of SVD events, and for the extraction of fundamental information from SVD hits. Useful algorithms that yield the absolute charge calibration of deposited energy and the determination of signal to noise thresholds for strip clustering have been developed and tested with data taken at the DESY' 16 test-beam. 


\section{References}

[1] A. Abashian et al. The Belle Detector. Nucl. Instrum. Meth., A479:117-232, 2002.

[2] K. Abe et al. Observation of Large CP Violation in the Neutral B Meson System. Physical Review Letters, 87(9):091802, August 2001.

[3] M. Kobayashi and T. Maskawa. CP-Violation in the Renormalizable Theory of Weak Interaction. Progress of Theoretical Physics, 49:652-657, February 1973.

[4] T. Abe et al. Belle II Technical Design Report. 2010.

[5] M. Friedl et al. The Belle II Silicon Vertex Detector. Nuclear Instruments and Methods in Physics Research Section A: Accelerators, Spectrometers, Detectors and Associated Equipment, 732:83 - 86, 2013. Vienna Conference on Instrumentation 2013.

[6] C. Patrignani et al. Review of Particle Physics. Chin. Phys., C40(10):100001, 2016.

[7] S. Agostinelli et al. GEANT4: A Simulation toolkit. Nucl. Instrum. Meth., A506:250-303, 2003. 